\title{
Discrete Wavelets and Multiresolution Analysis
}

\author{
Henk J. A. M. Heijmans
}

\begin{abstract}
In this paper we present an elementary discussion of the discrete wavelet transform. A major problem is formed by the construction of an orthonormal wavelet basis of the Hilbert space of square integrable functions. It is shown that the concept of a multiresolution analysis is very helpful to make such a construction. With every multiresolution analysis one can associate a father wavelet, the translates of which define an orthonormal system, and a mother wavelet which forms the basis for the discrete wavelet transform. This transform can be associated with two filtering operations and their adjoints; in practice these are used to get a pyramid-like decomposition of a signal. The abstract theory is illustrated by means of two concrete examples, the sinc-wavelet and the Meyer-wavelet.
\end{abstract}

\section{$\S 1$ Introduction}

For a long time the Fourier transform has been the most useful technique for the frequency analysis of a signal. However, due to the fact that sinusoids have an infinite support, such an approach has undesirable effects if one deals with signals which are localized in time or space (speech, imagery). So there is definitely a need for transforms which are not only localized in frequency but also in space. As an instance of such a transform we discuss the discrete wavelet transform. This is a mapping $T: L^{2}(\mathbb{R}) \rightarrow \ell^{2}\left(\mathbb{Z}^{2}\right)$ of the form

$$
(T f)_{n, k}=\left\langle f, \psi_{n, k}\right\rangle=\int_{\mathbf{R}} f(x) \psi_{n, k}(x) d x
$$

where $\psi_{n, k}$ is of the form

$$
\psi_{n, k}(x)=a^{-\frac{n}{2}} \psi\left(a^{-n} x-k b\right)
$$


and where the function $\psi$, known as the mother wavelet, satisfies

$$
\int_{\mathbf{R}} \psi(x) d x=0 \text {. }
$$

Note that as $n$ approaches $-\infty$, the coefficients $c_{k}^{n}(f)=\left\langle f, \psi_{n, k}\right\rangle$ express the highfrequency content of $f$ in a small neighbourhood of $k a^{n} b$, that is, they represent characteristics of the signal present at a smaller and smaller scale.

The linear operator $T$ has a bounded inverse on its range if

$$
A\|f\|^{2} \leq\|T f\|^{2} \leq B\|f\|^{2},
$$

for some constants $0<A \leq B<\infty$. If this condition holds then the set $\left\{\psi_{n, k} \mid\right.$ $n, k \in \mathbb{Z}\}$ is called a frame. In that case $f$ can be reconstructed from the wavelet coefficients $\left\langle f, \psi_{n, k}\right\rangle$; (see [4]).

In this paper we are particularly interested in the case where the $\psi_{n, k}$ constitute an orthonormal basis of $L^{2}(\mathbb{R})$. Furthermore we deal exclusively with the case where $a=2$ and $b=1$.

\section{Example 1.1. The Haar wavelet basis.}

The prototype example of a wavelet transform, which we consider at several instances in this paper, is given by the so-called Haar wavelet basis. Here $\psi$ equals

$$
\psi(x)= \begin{cases}1, & 0 \leq x<\frac{1}{2} \\ -1, & \frac{1}{2} \leq x<1 \\ 0, & \text { otherwise. }\end{cases}
$$

Note that $\int_{\mathbf{R}} \psi(x) d x=0$. The functions $\psi_{n, k}, n, k \in \mathbb{Z}$, given by $\psi_{n, k}(x)=$ $2^{-\frac{n}{2}} \psi\left(2^{-n} x-k\right)$ define an orthonormal basis of $L^{2}(\mathbb{R})$ called the Haar basis.
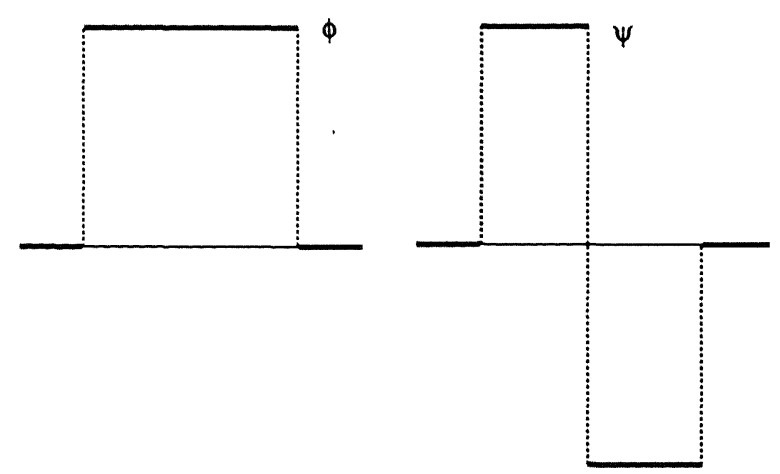

Figure 1. The Haar wavelet basis: the scaling function $\phi$ (left; see Section 3 for a definition) and the wavelet $\psi$ (right). 
Recall that the Fourier transform of $f$ is defined as

$$
\widehat{f}(\xi)=\int_{\mathbf{R}} e^{-i x \xi} f(x) d x
$$

We denote by $L_{P}^{2}$ the functions on $\mathbb{R}$ which are $P$-periodic and which satisfy $\int_{0}^{P}|f(x)|^{2} d x<\infty$. Throughout this paper we shall always use the notion of convergence of a series in the sense of "unconditional convergence".

In Section 2 we present a definition of multiresolution analysis. This notion underlies the description of the discrete wavelet transform and the construction of a mother wavelet. In Section 3 we use Fourier analysis to show that we can associate a scaling function (or father wavelet) $\phi$ to a given multiresolution analysis. This scaling function is determined by a sequence of coefficients $h_{k}$. In Section 4 we formulate conditions on $h_{k}$ which guarantee the existence of a scaling function and we show, again by Fourier analysis, how this scaling function can be computed. Then, in Section 5, we compute the mother wavelet $\psi$ which forms the basic entity for the discrete wavelet transform. In Section 6 we show that the coefficients in the discrete wavelet transform derive from two filtering operations and their adjoints. Finally, in Section 7 we apply our results to two concrete examples, namely the sinc-wavelet and the Meyer-wavelet.

\section{$\S 2$ Multiresolution analysis}

Now the question arises how one can construct an (orthonormal) wavelet basis. A systematic way to do this is by means of a so-called multiresolution analysis. We will introduce this concept, which is originally due to Mallat and Meyer, in our following definition. Recall that a system $\left\{\phi_{k} \mid k \in \mathbb{Z}\right\}$ is called a Riesz basis if it is obtained from an orthonormal basis by means of a bounded invertible operator [10].

Definition 2.1. A multiresolution analysis of $L^{2}(\mathbb{R})$ is a sequence of closed subspaces $\cdots, V_{-1}, V_{0}, V_{1}, V_{2}, \cdots$ such that

(M1) $\quad V_{n} \subset V_{n-1}, n \in \mathbb{Z}$.

(M2) $\bigcup_{n=-\infty}^{\infty} V_{n}$ is dense in $L^{2}$ and $\bigcap_{n=-\infty}^{\infty} V_{n}=\{0\}$.

(M3) $f(x) \in V_{n} \Longleftrightarrow f(2 x) \in V_{n-1}$.

(M4) $f(x) \in V_{0} \Longleftrightarrow f(x-k) \in V_{0}$, for all $k \in \mathbb{Z}$.

(M5) There exists a function $g \in V_{0}$ such that the collection $g(\cdot-k)$, $k \in \mathbb{Z}$ is a Riesz basis for $V_{0}$.

We point out that the function $g$ in (M5) is not unique. Let $P_{n}$ be the orthogonal projection of $L^{2}(\mathbb{R})$ onto $V_{n}$. Then condition (M1) can be restated in terms of $P_{n}$ as follows:

$$
P_{n} P_{m}=P_{m} P_{n}=P_{n}, \quad m \leq n .
$$


Furthermore, condition (M2) means that

$$
\lim _{n \rightarrow-\infty} P_{n} f=f, \quad \lim _{n \rightarrow \infty} P_{n} f=0
$$

for every $f \in L^{2}(\mathbb{R})$. Finally, condition (M3) can be expressed as

$$
P_{n-1}=D_{\frac{1}{2}} P_{n} D_{2}
$$

where $D_{a}$ is the dilation operator given by $\left(D_{a} f\right)(x)=|a|^{-\frac{1}{2}} f(x / a)$.

\section{Example 2.2. The Haar wavelet basis}

Take $V_{n}$ to be the space of functions in $L^{2}(\mathbb{R})$ which are piecewise constant on the intervals $\left[2^{n} k, 2^{n}(k+1)\right)$. It is obvious that the conditions (M1)-(M5) are satisfied if we take for $g$ the characteristic function of the interval $[0,1)$. Notice that in this case the functions $g(\cdot-k), k \in \mathbb{Z}$, constitute an orthonormal basis of $V_{0}$. It is easy to check that the projection $P_{n}$ is given by

$$
\left(P_{n} f\right)(x)=2^{-n} \int_{2^{n} k}^{2^{n}(k+1)} f(y) d y, \quad x \in\left[2^{n} k, 2^{n}(k+1)\right) .
$$

In this example the elements of the subspaces $V_{n}$ are only piecewise continuous, and as a consequence the projection $P_{n} f$ converges to $f$ very slowly as $n \rightarrow \infty$. To obtain faster approximations one has to assume some additional regularity for the functions in $V_{n}$, or in other words, for the function $g$. We give a formal definition.

Definition 2.3. A function $f \in L^{2}(\mathbb{R})$ is said to be regular if it is continuously differentiable and satisfies

$$
|f(x)| \leq \frac{C}{1+x^{2}}, \quad\left|f^{\prime}(x)\right| \leq \frac{C}{1+x^{2}},
$$

for some constant $C>0$ and every $x \in \mathbb{R}$. A multiresolution analysis is said to be regular if the function $g$ in (M5) is regular.

\section{Example 2.4. Higher order splines}

The space $V_{0}$ in the example of the Haar basis consists of 1'st order splines. In this example we consider splines of order $r \geq 1$. Let $V_{0}=\left\{f \in L^{2}(\mathbb{R}) \mid f \in C^{r-1}\right.$ and $f$ is a polynomial of degree $\leq r$ on every integer interval $[k, k+1]\}$. If, for instance, $r=1$ then $V_{0}$ consists of piecewise affine functions. Defining the $V_{n}$ by dilation of $V_{0}$, one easily derives that (M1)-(M4) are satisfied. Let $\chi$ be the characteristic function of the interval $[0,1]$ and define the $r$-fold convolution $\chi^{* r}=\chi * \chi * \cdots * \chi$. One can show that $g=\chi^{*(r+1)}$ satisfies (M5). The functions $\chi(\cdot-k)$ form an 
orthonormal basis for $V_{0}$ in the case $r=0$. This, however, is no longer true if $r \geq 1$. Note that if $r=1$, the function $g=\chi^{* 2}$ is given by

$$
g(x)= \begin{cases}x, & 0 \leq x \leq 1 \\ 2-x, & 1 \leq x \leq 2 \\ 0, & \text { outside }[0,2]\end{cases}
$$

Using that $\chi(x)=\chi(2 x)+\chi(2 x-1)$ we find by a straightforward computation that $g=\chi^{* 2}$ satisfies

$$
g(x)=\frac{1}{2} g(2 x)+g(2 x-1)+\frac{1}{2} g(2 x-2)
$$

(see Figure 2 below).

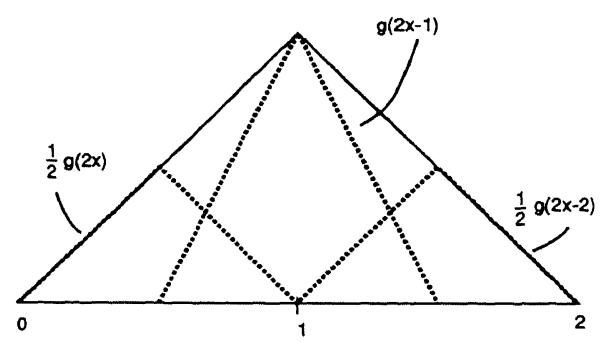

Figure 2. The scaling function $g=\chi^{* 2}$ for second order splines.

Similar expressions can be derived for $\chi^{* r}$ if $r \geq 1$.

\section{$\S 3$ Orthonormal basis of multiresolution analysis}

In the last example we have seen that for splines of order $\geq r$ the basis elements $\chi^{*(r+1)}(\cdot-k)$ are not orthonormal. In this section we will show, using standard Fourier techniques, that for every multiresolution analysis $\left\{V_{n}\right\}$ one can always find a function $\phi \in V_{0}$ such that the functions $\phi(\cdot-k), k \in \mathbb{Z}$ form an orthonormal basis. In that case $\phi$ will be called the scaling function or also the father wavelet of the multiresolution analysis $\left\{V_{n}\right\}$.

Assume that $V_{0} \subset L^{2}(\mathbb{R})$ is such that (M4)-(M5) hold. If $f \in V_{0}$ then it can be decomposed as

$$
f(x)=\sum_{k=-\infty}^{\infty} a_{k} g(x-k)
$$


where $a=\left(a_{k}\right) \in \ell^{2}(\mathbb{Z})$. Now we take the Fourier transform at both sides, change summation and integration (which is allowed since the Fourier transform is a continuous operator) and arrive at

$$
\widehat{f}(\xi)=\sum_{k=-\infty}^{\infty} a_{k} e^{-i k \xi} \widehat{g}(\xi) .
$$

Let $A_{f} \in L_{2 \pi}^{2}$ be given by $A_{f}(\xi)=\sum_{k=-\infty}^{\infty} a_{k} e^{-i k \xi}$. The next lemma shows that we can decompose the infinite sum representing $\widehat{f}(\xi)$.

Lemma 3.1. Let $a=\left(a_{k}\right) \in \ell^{2}(\mathbb{Z})$ and $g \in L^{2}(\mathbb{R})$. Then

$$
\sum_{k=-\infty}^{\infty} a_{k} e^{-i k \xi} \widehat{g}(\xi)=\left(\sum_{k=-\infty}^{\infty} a_{k} e^{-i k \xi}\right) \cdot \widehat{g}(\xi)
$$

where convergence is taken in the $L^{2}$-norm and the first factor at the right-hand-side is interpreted as an element of $L_{2 \pi}^{2}$.

Proof: In the proof we use the following result twice. If $f_{n}$ is a sequence in $L^{2}(\Omega)$, where $\Omega$ is a measure space, and $f_{n} \rightarrow f$ as $n \rightarrow \infty$ in the $L^{2}$-norm, then there is a subsequence $\left\{n_{p}\right\}_{p}$ such that

$$
f_{n_{p}}(y) \rightarrow f(y), p \rightarrow \infty, \quad \text { for a.e. } y \in \Omega ;
$$

(see $\left[8\right.$, Thm 3.12]). We apply this to the sequence $\left\{\sum_{|k| \leq N} a_{k} e^{-i k \xi} \widehat{g}(\xi)\right\}_{N}$ and find that there is a subsequence $\left\{N_{p}\right\}$ such that

$$
\lim _{p \rightarrow \infty} \sum_{|k| \leq N_{p}} a_{k} e^{-i k \xi} \widehat{g}(\xi)=\sum_{k=-\infty}^{\infty} a_{k} e^{-i k \xi} \widehat{g}(\xi),
$$

for a.e. $\xi \in \mathbb{R}$. Now consider the term $\left(\sum_{|k| \leq N_{p}} a_{k} e^{-i k \xi}\right) \widehat{g}(\xi)$ at the left handside. We know that $\sum_{|k| \leq N_{p}} a_{k} e^{-i k}$. converges to $\sum_{k=-\infty}^{\infty} a_{k} e^{-i k}$ in the $L_{2 \pi}^{2}$-norm. Hence there is a subsequence $\left\{p_{j}\right\}_{j}$ such that

$$
\lim _{j \rightarrow \infty} \sum_{|k| \leq N_{p_{j}}} a_{k} e^{-i k \xi}=\sum_{k=-\infty}^{\infty} a_{k} e^{-i k \xi},
$$

for a.e. $\xi \in \mathbb{R}$. This proves the result.

From this lemma we may conclude that $f \in V_{0}$ if and only if

$$
\widehat{f}(\xi)=A_{f}(\xi) \widehat{g}(\xi)
$$


for some $A_{f} \in L_{2 \pi}^{2}$. The norm of $f$ is given by

$$
\begin{aligned}
\|f\|^{2} & =\frac{1}{2 \pi} \int_{\mathbb{R}}|\widehat{f}(\xi)|^{2} d \xi \\
& =\frac{1}{2 \pi} \sum_{k=-\infty}^{\infty} \int_{k \cdot 2 \pi}^{(k+1) \cdot 2 \pi}\left|A_{f}(\xi)\right|^{2}|\widehat{g}(\xi)|^{2} d \xi \\
& =\frac{1}{2 \pi} \int_{0}^{2 \pi}\left|A_{f}(\xi)\right|^{2}\left(\sum_{k=-\infty}^{\infty}|\widehat{g}(\xi+2 k \pi)|^{2}\right) d \xi
\end{aligned}
$$

where we have used that $A_{f}$ is $2 \pi$-periodic. Putting

$$
\Gamma(\xi)=\left(\sum_{k=-\infty}^{\infty}|\widehat{g}(\xi+2 k \pi)|^{2}\right)^{\frac{1}{2}}
$$

we get that

$$
\|f\|^{2}=\frac{1}{2 \pi} \int_{0}^{2 \pi}\left|A_{f}(\xi)\right|^{2} \Gamma(\xi)^{2} d \xi
$$

Lemma 3.2. Let $V_{0} \subset L^{2}(\mathbb{R})$ and $g \in V_{0}$ be such that (M4)-(M5) hold. Then there exist $C_{1}, C_{2}>0$ such that

$$
C_{1} \leq \Gamma(\xi) \leq C_{2}, \quad \xi \in \mathbb{R}
$$

The proof can be found in [7, Thm II.1, p. 27].

We define $U: V_{0} \rightarrow L^{2}[0,2 \pi]$ as the bounded linear operator which maps the function $g(\cdot-k)$ to $(2 \pi)^{-\frac{1}{2}} e^{-i k \cdot} \Gamma$, or more generally

$$
(U f)(\xi)=\frac{1}{\sqrt{2 \pi}} A_{f}(\xi) \Gamma(\xi)
$$

It is obvious that $U$ is injective. To show that $U$ is surjective take an arbitrary $h \in L^{2}[0,2 \pi]$. Then, thanks to Lemma $3.2, h / \Gamma \in L^{2}[0,2 \pi]$ and so there exists a sequence $\left(a_{k}\right) \in \ell^{2}(\mathbb{Z})$ such that

$$
\sqrt{2 \pi} \frac{h(\xi)}{\Gamma(\xi)}=\sum_{k=-\infty}^{\infty} a_{k} e^{-i k \xi}
$$

Then

$$
U\left(\sum_{k=-\infty}^{\infty} a_{k} g(\cdot-k)\right)=\frac{1}{\sqrt{2 \pi}} \sum_{k=-\infty}^{\infty} a_{k} e^{-i k \xi} \Gamma(\xi)=h(\xi)
$$


Thus $U$ defines an isomorphism. From (3.4) we conclude that $U$ is also an isometry, i.e., $\|U f\|=\|f\|$ for every $f \in V_{0}$. Thus we arrive at the following result.

Lemma 3.3. $U$ is an isometric isomorphism.

Assume that $f \in V_{0}$. Then there is a sequence $\left(a_{k}\right) \in \ell^{2}(\mathbb{Z})$ such that $f=$ $\sum_{k=-\infty}^{\infty} a_{k} g(\cdot-k)$. Then

$$
(U f)(\xi)=\frac{1}{\sqrt{2 \pi}} \sum_{k=-\infty}^{\infty} a_{k} e^{-i k \xi} \Gamma(\xi)
$$

Furthermore, $\hat{f}(\xi)=\sum_{k=-\infty}^{\infty} a_{k} e^{-i k \xi} \widehat{g}(\xi)$. Combination of both expressions yields that

$$
(U f)(\xi)=\frac{1}{\sqrt{2 \pi}} \frac{\hat{f}(\xi)}{\widehat{g}(\xi)} \Gamma(\xi) .
$$

Note that

$$
U f(\cdot-k)=e^{-i k \cdot} U f
$$

for every $f \in V_{0}$. Assume that $\phi(\cdot-k), k \in \mathbb{Z}$, is an orthonormal basis of $V_{0}$. Then, since $U$ is an isometric isomorphism, $U \phi(\cdot-k)$ is an orthonormal basis of $L^{2}[0,2 \pi]$. Let $e_{k} \in L^{2}[0,2 \pi]$ be given by

$$
e_{k}=U \phi(\cdot-k)=e^{-i k \cdot} U \phi
$$

Then

$$
\left\langle e_{k}, e_{l}\right\rangle=\int_{0}^{2 \pi} e^{-i(k-l) \xi}|U \phi(\xi)|^{2} d \xi
$$

and $\left\langle e_{k}, e_{l}\right\rangle=\delta_{k l}$ if $|U \phi(\xi)|=1 / \sqrt{2 \pi}$ a.e. Using (3.7) we obtain that a solution is given by $\widehat{\phi}(\xi)=\widehat{g}(\xi) / \Gamma(\xi)$.

Theorem 3.4. Let $V_{0} \subset L^{2}(\mathbb{R})$ and let $g \in V_{0}$ be such that (M4)-(M5) hold.

(a) If $\phi \in L^{2}(\mathbb{R})$ is defined by

$$
\widehat{\phi}(\xi)=\frac{\widehat{g}(\xi)}{\Gamma(\xi)}
$$

where $\Gamma(\xi)$ is given by (3.3), then the functions $\phi(\cdot-k)$ form an orthonormal basis of $V_{0}$.

(b) If $\sigma \in L^{\infty}(\mathbb{R})$ is a $2 \pi$-periodic function with $|\sigma(\xi)|=1$ a.e., and if $\theta$ is defined by $\widehat{\theta}(\xi)=\sigma(\xi) \widehat{\phi}(\xi)$, then $\theta \in V_{0}$ and $\theta(\cdot-k), k \in \mathbb{Z}$ is an orthonormal basis of $V_{0}$.

Conversely, if $\theta \in V_{0}$ is such that $\|\theta\|=1$ and the functions $\theta(\cdot-k)$ are orthogonal, then $\theta(\cdot-k), k \in \mathbb{Z}$, is an orthonormal basis of $V_{0}$ and $\widehat{\theta}(\xi)=\sigma(\xi) \widehat{\phi}(\xi)$ 
where $\sigma \in L^{\infty}(\mathbb{R})$ is a $2 \pi$-periodic function with $|\sigma(\xi)|=1$ a.e.

(c) Let $\phi \in V_{0}$ be such that the system $\phi(\cdot-k), k \in \mathbb{Z}$, is orthonormal. Then

$$
\sum_{k=-\infty}^{\infty}|\widehat{\phi}(\xi+2 k \pi)|^{2}=1 \quad \text { a.e. }
$$

Proof: (a) and the first assertion in (b) follow from the arguments given above. To prove the second assertion in (b) assume that $\|\theta\|=1$ and $\theta(\cdot-k)$ is orthogonal. Define $\sigma=\sqrt{2 \pi} U \theta$, then $U \theta(\cdot-k)=(2 \pi)^{-\frac{1}{2}} e^{-i k \cdot} \sigma$. Since $U$ is an isometry we get that $\|\sigma\|=\sqrt{2 \pi}$. Furthermore $\left\{e^{-i k \cdot} \sigma \mid k \in \mathbb{Z}\right\}$ is an orthogonal system and hence

$$
\int_{0}^{2 \pi} \sigma(\xi) \bar{\sigma}(\xi) e^{i k \xi} d \xi=0 \quad \text { if } k \neq 0
$$

yielding that $|\sigma(\xi)|^{2}$ is a constant a.e. In combination with $\|\sigma\|=\sqrt{2 \pi}$ this yields that $|\sigma(\xi)|=1$ a.e. We may conclude that the family $\left\{(2 \pi)^{-\frac{1}{2}} e^{-i k \cdot} \sigma \mid k \in \mathbb{Z}\right\}$ is an orthonormal basis of $L^{2}[0,2 \pi]$ and hence that $\{\theta(\cdot-k) \mid k \in \mathbb{Z}\}$ is an orthonormal basis of $V_{0}$. Finally

$$
\sigma=\sqrt{2 \pi} U \theta=\frac{\widehat{\theta} \Gamma}{\widehat{g}}=\frac{\widehat{\theta}}{\widehat{\phi}},
$$

whence the conclusion follows.

(c) If $\phi(\cdot-k)$ are orthonormal, then

$$
\begin{aligned}
\delta_{m 0} & =\int_{\mathbf{R}} \phi(x) \bar{\phi}(x-m) d x=\frac{1}{2 \pi} \int_{\mathbf{R}} e^{-i m \xi}|\widehat{\phi}(\xi)|^{2} d \xi \\
& =\frac{1}{2 \pi} \int_{0}^{2 \pi} e^{-i m \xi}\left(\sum_{k=-\infty}^{\infty}|\widehat{\phi}(\xi+2 k \pi)|^{2}\right) d \xi
\end{aligned}
$$

From this, the assertion follows.

\section{$\S 4$ Construction of a scaling function}

A crucial question is how one can find a function $\phi$ such that the translates $\phi(\cdot-k)$ are orthonormal and such that the dilation $D_{2} \phi$ lies in the linear space spanned by these translates. We start with a lemma.

Lemma 4.1. Let $g$ be a regular function and let the kernel $K$ be given by $K(x, y)=$ $\sum_{k=-\infty}^{\infty} g(x-k) \bar{g}(y-k)$. Let $T_{\lambda}: L^{2}(\mathbb{R}) \rightarrow L^{2}(\mathbb{R})$ be the integral operator

$$
\left(T_{\lambda} f\right)(x)=\lambda \int K(\lambda x, \lambda y) f(y) d y .
$$


The following assertions are equivalent:

(i) $\quad \lim _{\lambda \rightarrow \infty}\left\|T_{\lambda} f-f\right\|_{2}=0$, for $f \in L^{2}(\mathbb{R})$.

(ii) $\int K(x, y) d y=1$ for a.e $x \in \mathbb{R}$.

The proof can be found in [5, Lemma 1, p. 74]; (see also [7, Lemma II.13, p. 42]).

Assume that $V_{n}, n \in \mathbb{Z}$, defines a regular multiresolution analysis of $L^{2}(\mathbb{R})$ generated by the scaling function $\phi$, and that $\{\phi(\cdot-k) \mid k \in \mathbb{Z}\}$ is an orthonormal family. The function $\phi\left(\frac{1}{2} \cdot\right)$ lies in $V_{1}$ and hence in $V_{0}$, so we can write

$$
\phi\left(\frac{x}{2}\right)=\sqrt{2} \sum_{k=-\infty}^{\infty} h_{k} \phi(x-k)
$$

where

$$
h_{k}=\frac{1}{\sqrt{2}} \int_{\mathbf{R}} \phi\left(\frac{x}{2}\right) \bar{\phi}(x-k) d x .
$$

Since $\phi$ is regular we find that

$$
\left|h_{k}\right|=\mathcal{O}\left(\frac{1}{1+k^{2}}\right), k \rightarrow \infty
$$

Taking the Fourier transform of (4.1) at both sides we get that

$$
\widehat{\phi}(2 \xi)=H(\xi) \widehat{\phi}(\xi)
$$

where

$$
H(\xi)=\frac{1}{\sqrt{2}} \sum_{k=-\infty}^{\infty} h_{k} e^{-i k \xi} .
$$

In the previous section (cf. Theorem $3.4(\mathrm{c})$ ) we have seen that

$$
\sum_{k=-\infty}^{\infty}|\widehat{\phi}(\xi+2 k \pi)|^{2}=1
$$

In combination with (4.4) this yields that

$$
1=\sum_{k=-\infty}^{\infty}|\widehat{\phi}(2 \xi+2 k \pi)|^{2}=\sum_{k=-\infty}^{\infty}|H(\xi+k \pi)|^{2}|\widehat{\phi}(\xi+k \pi)|^{2}
$$

Since $H$ is $2 \pi$-periodic we find that

$$
1=|H(\xi)|^{2} \sum_{k=-\infty}^{\infty}|\widehat{\phi}(\xi+2 k \pi)|^{2}+|H(\xi+\pi)|^{2} \sum_{k=-\infty}^{\infty}|\widehat{\phi}(\xi+\pi+2 k \pi)|^{2}
$$


and hence that

$$
|H(\xi)|^{2}+|H(\xi+\pi)|^{2}=1 .
$$

Restated in terms of $h_{k}$ this condition reads

$$
\sum_{n=-\infty}^{\infty} h_{n-2 k} \bar{h}_{n-2 l}=\delta_{k l} .
$$

Lemma 4.2. Under the given assumptions

$$
H(0)=\frac{1}{\sqrt{2}} \sum_{k=-\infty}^{\infty} h_{k}=1
$$

This result will follow from (4.4) if we can show that $\widehat{\phi}(0) \neq 0$. Actually we show below that $|\widehat{\phi}(0)|=1$. We consider the projection operator $P_{n}$ on $V_{n}$. Let $P_{0}$ be the projection operator on $V_{0}$. One easily sees that the following intertwining diagram is valid; (see (2.1)). Here $D_{a}$ is the dilation operator given by $\left(D_{a} f\right)(x)=$ $|a|^{-\frac{1}{2}} f(x / a)$.

$$
\begin{array}{ccc}
L^{2}(\mathbb{R}) & \stackrel{P_{n}}{\longrightarrow} & V_{n} \\
D_{\frac{1}{2}} \downarrow\left\lceil D_{2}\right. & & D_{\frac{1}{2}} \downarrow\left\lceil D_{2}\right. \\
L^{2}(\mathbb{R}) & \stackrel{P_{n-1}}{\longrightarrow} & V_{n-1}
\end{array}
$$

In particular we have

$$
P_{n}=D_{2^{n}} P_{0} D_{2^{-n}}
$$

Now $P_{0} f=\sum_{k=-\infty}^{\infty}\langle f, \phi(\cdot-k)\rangle \phi(\cdot-k)$, which yields that

$$
P_{0} f(x)=\int_{\mathbf{R}} K(x, y) f(y) d y
$$

where

$$
K(x, y)=\sum_{k=-\infty}^{\infty} \phi(x-k) \bar{\phi}(y-k) .
$$

Since $\phi$ is regular we can show that

$$
|K(x, y)| \leq \frac{C}{1+|x-y|^{2}}
$$

for some constant $C>0$. From (4.9) we conclude that $P_{n}$ is the integral operator with kernel $2^{-n} K\left(2^{-n} x, 2^{-n} y\right)$. Since $\phi$ generates a multiresolution analysis of 
$L^{2}(\mathbb{R})$ we conclude from (M2) that $P_{n} f \rightarrow f$ as $n \rightarrow-\infty$ with respect to the strong operator topology. Applying Lemma 4.1 we get that $\int_{\mathbf{R}} K(x, y) d y=1$ for a.e. $x$, that is,

$$
\sum_{k=-\infty}^{\infty} \phi(x-k) \int_{\mathbf{R}} \bar{\phi}(y-k) d y=\overline{\widehat{\phi}}(0) \sum_{k=-\infty}^{\infty} \phi(x-k)=1 .
$$

Integrating this expression over $[0,1]$ we find that

$$
|\widehat{\phi}(0)|^{2}=1 \text {. }
$$

Now, by substituting $\xi=0$ in (4.4) the result stated in Lemma 4.2 follows.

Remark. 4.3. From $H(\pi)=0$ it follows that $\sum_{k=-\infty}^{\infty}(-1)^{k} h_{k}=0$, that is

$$
\sum_{k=-\infty}^{\infty} h_{2 k}=\sum_{k=-\infty}^{\infty} h_{2 k+1}=\frac{1}{\sqrt{2}}
$$

If $\left|h_{k}\right|$ decreases fast enough, in particular, if $h_{k}=0$ for $|k|$ large enough, one can show that

$$
H(\xi)=\left(1+e^{-i \xi}\right) F(\xi)
$$

where $F(\xi)=\sum_{k=-\infty}^{\infty} f_{k} e^{-i k \xi}$ and $\left(f_{k}\right) \in \ell^{2}$.

In the remainder of this section we show that conditions (4.7)-(4.8) along with some other (technical) conditions, mainly concerning regularity, yield a multiresolution analysis.

Theorem 4.4. Let $\left(h_{k}\right) \in \ell^{2}(\mathbb{Z})$ be a sequence which satisfies

$$
\begin{gathered}
\left|h_{k}\right|=\mathcal{O}\left(\frac{1}{1+k^{2}}\right), \quad k \rightarrow \infty \\
\sum_{k=-\infty}^{\infty} h_{k}=\sqrt{2} \quad \text { and } \quad \sum_{n=-\infty}^{\infty} h_{n-2 k} \bar{h}_{n-2 l}=\delta_{k l} .
\end{gathered}
$$

Define $H(\xi)=\frac{1}{\sqrt{2}} \sum_{k=-\infty}^{\infty} h_{k} e^{-i k \xi}$ and assume that

$$
H(\xi) \neq 0 \text { on }\left[-\frac{\pi}{2}, \frac{\pi}{2}\right] .
$$

Then the infinite product $\prod_{k=1}^{\infty} H\left(2^{-k} \xi\right)$ defines an $L^{2}$-function. Let $\phi \in L^{2}(\mathbb{R})$ be the function with Fourier transform

$$
\widehat{\phi}(\xi)=\prod_{k=1}^{\infty} H\left(2^{-k} \xi\right)
$$


Then $\{\phi(\cdot-k) \mid k \in \mathbb{Z}\}$ defines an orthonormal system. Let $V_{0}$ be the subspace of $L^{2}(\mathbb{R})$ spanned by this system and let $V_{n}=D_{2^{n}} V_{0}$ for $n \in \mathbb{Z}$. If $\phi$ is regular then the $V_{n}$ define a regular multiresolution of $L^{2}(\mathbb{R})$.

The proof of this theorem consists of several steps. We start with a lemma due to Daubechies [1, Lemma 3.1, p. 948].

Lemma 4.5. Let $\left(h_{k}\right)$ be a sequence with $\sum_{k=-\infty}^{\infty} h_{k}=\sqrt{2}$ and let $H(\xi)=$ $2^{-\frac{1}{2}} \sum_{k=-\infty}^{\infty} h_{k} e^{-i k \xi}$. Assume that for some $\varepsilon>0$ we have

$$
\sum_{k=-\infty}^{\infty}\left|h_{k}\right| \cdot|k|^{\varepsilon}<\infty
$$

Then $\prod_{k=-\infty}^{\infty} H\left(2^{-k} \xi\right)$ converges pointwise for every $\xi \in \mathbb{R}$, and the convergence is uniform on compact sets.

Proof: It is easily seen that

$$
H(\xi)=1+\sqrt{2} \sum_{k=-\infty}^{\infty} h_{k} \cdot\left(\frac{e^{-i k \xi}-1}{2}\right),
$$

and hence

$$
|H(\xi)-1| \leq \sqrt{2} \sum_{k=-\infty}^{\infty}\left|h_{k}\right| \cdot\left|\sin \frac{k \xi}{2}\right| .
$$

We assume without loss of generality that $\varepsilon \leq 1$ in (4.18). There is a $C>0$ such that $|\sin \theta| \leq C|\theta|^{\varepsilon}$ for every $\theta \in \mathbb{R}$, which yields that

$$
|H(\xi)-1| \leq \sqrt{2} C \sum_{k=-\infty}^{\infty}\left|h_{k}\right| \cdot\left|\frac{1}{2} k \xi\right|^{\varepsilon} \leq C^{\prime}|\xi|^{\varepsilon} .
$$

Substituting $2^{-k} \xi$ we find that

$$
\left|H\left(2^{-k} \xi\right)-1\right| \leq C^{\prime} 2^{-k \varepsilon}|\xi|^{\varepsilon} .
$$

From this the convergence of the infinite product $\prod_{k=1}^{\infty} H\left(2^{-k} \xi\right)$ follows immediately.

We put

$$
H_{\infty}(\xi)=\prod_{k=1}^{\infty} H\left(2^{-k} \xi\right) .
$$

Next we show that this infinite product defines an $L^{2}$-function. Thereto we introduce the following notation: we put

$$
M(\xi)=|H(\xi)|^{2}
$$


Furthermore, we let for $k \geq 1, M_{k}$ be the continuous function

$$
M_{k}(\xi)= \begin{cases}0, & \text { if }|\xi|>2^{k} \pi \\ M\left(\frac{1}{2} \xi\right) M\left(\frac{1}{4} \xi\right) \cdots M\left(2^{-k} \xi\right) & \text { if }|\xi| \leq 2^{k} \pi\end{cases}
$$

Lemma 4.6. For every $k=1,2, \cdots$

$$
\int_{\mathbf{R}} M_{k}(\xi) e^{i m \xi} d \xi= \begin{cases}2 \pi, & m=0 \\ 0, & m \neq 0\end{cases}
$$

Proof: Using that $M(\xi)+M(\xi+\pi)=1$ it follows immediately that

$$
\int_{\mathbf{R}} M_{1}(\xi) e^{i m \xi} d \xi=\int_{-2 \pi}^{2 \pi} M\left(\frac{1}{2} \xi\right) e^{i m \xi} d \xi=2 \pi \delta_{m 0}
$$

Furthermore,

$$
\begin{aligned}
\int_{\mathbf{R}} M_{2}(\xi) e^{i m \xi} d \xi & =\int_{-4 \pi}^{4 \pi} M\left(\frac{1}{2} \xi\right) M\left(\frac{1}{4} \xi\right) e^{i m \xi} d \xi \\
& =\int_{-4 \pi}^{0} M\left(\frac{1}{2} \xi\right) M\left(\frac{1}{4} \xi\right) e^{i m \xi} d \xi+\int_{0}^{4 \pi} M\left(\frac{1}{2} \xi\right) M\left(\frac{1}{4} \xi\right) e^{i m \xi} d \xi \\
& =\int_{0}^{4 \pi} M\left(\frac{1}{2} \xi+2 \pi\right) M\left(\frac{1}{4} \xi+\pi\right) e^{i m \xi} d \xi \\
& +\int_{0}^{4 \pi} M\left(\frac{1}{2} \xi\right) M\left(\frac{1}{4} \xi\right) e^{i m \xi} d \xi \\
& =\int_{0}^{4 \pi} M\left(\frac{1}{2} \xi\right)\left[M\left(\frac{1}{4} \xi+\pi\right)+M\left(\frac{1}{4} \xi\right)\right] e^{i m \xi} d \xi \\
& =\int_{0}^{4 \pi} M\left(\frac{1}{2} \xi\right) e^{i m \xi} d \xi \\
& =\int_{-2 \pi}^{2 \pi} M\left(\frac{1}{2} \xi\right) e^{i m \xi} d \xi
\end{aligned}
$$

where we have used that $M\left(\frac{1}{2} \cdot\right)$ is $4 \pi$-periodic in the last equality. This shows that

$$
\int_{\mathbf{R}} M_{2}(\xi) e^{i m \xi} d \xi=\int_{\mathbf{R}} M_{1}(\xi) e^{i m \xi} d \xi=2 \pi \delta_{m 0}
$$

The same relations can be established for $k>2$, and we conclude that

$$
\int_{\mathbf{R}} M_{k}(\xi) e^{i m \xi} d \xi=\int_{\mathbf{R}} M_{1}(\xi) e^{i m \xi} d \xi=2 \pi \delta_{m 0}
$$


This concludes the proof.

We are now ready to finish the proof of Theorem 4.4.

Proof of Theorem 4.4

Since $M(\xi) \leq 1$, the sequence $M_{k}(\xi)$ converges as $k \rightarrow \infty$,

$$
M_{\infty}(\xi)=\lim _{k \rightarrow \infty} M_{k}(\xi)=\prod_{k=1}^{\infty} M\left(2^{-k} \xi\right)=\left|H_{\infty}(\xi)\right|^{2}
$$

Fatou's lemma [8] gives

$$
\int_{\mathbf{R}} M_{\infty}(\xi) \leq \lim _{k \rightarrow \infty} \int_{\mathbf{R}} M_{k}(\xi) d \xi=2 \pi
$$

from which we conclude that $H_{\infty}$ is an element of $L^{2}(\mathbb{R})$. Let $\phi \in L^{2}(\mathbb{R})$ be the function which has $H_{\infty}$ as its Fourier transform, that is,

$$
\widehat{\phi}(\xi)=\prod_{k=1}^{\infty} H\left(2^{-k} \xi\right)
$$

We show that the system $\{\phi(\cdot-k) \mid k \in \mathbb{Z}\}$ is orthonormal. From Parseval's formula we find that

$$
\int_{\mathbf{R}} \phi(x) \bar{\phi}(x-k) d x=\frac{1}{2 \pi} \int_{\mathbf{R}} e^{i k \xi}|\widehat{\phi}(\xi)|^{2} d \xi=\frac{1}{2 \pi} \int_{\mathbf{R}} e^{i k \xi} M_{\infty}(\xi) d \xi
$$

Suppose we can show that there exists an $L^{1}$-function $F$ such that

$$
0 \leq M_{k}(\xi) \leq F(\xi), \quad \text { for } \xi \in \mathbb{R}
$$

Then, by Lebesgue's dominated convergence theorem and Lemma 4.6 we derive that

$$
2 \pi \delta_{m 0}=\lim _{k \rightarrow \infty} \int_{\mathbf{R}} M_{k}(\xi) e^{i m \xi} d \xi=\int_{\mathbf{R}} M_{\infty}(\xi) e^{i m \xi} d \xi
$$

whence the assertion follows. So it remains to establish (4.23) for some $F \in L^{1}(\mathbb{R})$. From the regularity condition (4.14) and the fact that $H(0)=1$ we conclude that $H(\xi)=1+\mathcal{O}(\xi)$ as $\xi \rightarrow 0$, and hence that $M(\xi)=1+\mathcal{O}(\xi)$ for $\xi \rightarrow 0$. Since

$$
M_{\infty}(\xi)=\exp \left\{\sum_{k=1}^{\infty} \log M\left(2^{-k} \xi\right)\right\}
$$


we find that

$$
M_{\infty}(\xi)=1+\mathcal{O}(\xi), \quad \xi \rightarrow 0 .
$$

In combination with $M_{\infty}(2 \xi)=M(\xi) M_{\infty}(\xi)=|H(\xi)|^{2} M_{\infty}(\xi)$ and the assumption (4.16) this implies that

$$
M_{\infty}(\xi) \geq c, \quad \xi \in[-\pi, \pi]
$$

for some $c>0$. For $|\xi| \leq 2^{k} \pi$ we have $M_{\infty}(\xi)=M_{k}(\xi) M_{\infty}\left(\xi / 2^{k}\right)$ and with (4.25) this yields that

$$
0 \leq M_{k}(\xi) \leq \frac{1}{c} M_{\infty}(\xi)
$$

for $|\xi| \leq 2^{k} \pi$. However, $M_{k}(\xi)=0$ for $|\xi| \geq 2^{k} \pi$ and therefore this inequality holds for every $\xi \in \mathbb{R}$. Thus $(4.23)$ holds with $F=c^{-1} M_{\infty}$. This shows that the $\phi(\cdot-k)$ define an orthonormal system.

We now assume in addition that $\phi$ is regular. Let $V_{0}$ be the vector space spanned by the $\phi(\cdot-k)$ and let $V_{n}$ be the spaces deriving from $V_{0}$ by dilation. We must show that the $V_{n}$ define a multiresolution analysis of $L^{2}(\mathbb{R})$. To prove (M1) it suffices to show that $V_{1} \subset V_{0}$. If $f \in V_{0}$ (resp. $\left.V_{1}\right)$ then $\widehat{f}(\xi)=A_{f}(\xi) \widehat{\phi}(\xi)$ (resp. $\widehat{f}(\xi)=A_{f}(2 \xi) \widehat{\phi}(2 \xi)$ ) for some $2 \pi$-periodic function $A_{f} \in L^{2}[0,2 \pi]$. From the expression (4.22) for $\widehat{\phi}$ we deduce that

$$
\widehat{\phi}(2 \xi)=H(\xi) \widehat{\phi}(\xi)
$$

where $H$ is $2 \pi$-periodic and $|H(\xi)| \leq 1$. From these observations it follows that $V_{1} \subset V_{0}$. Now let $P_{n}$ be the orthogonal projection on $V_{n}$. To prove (M2) we must show that

$$
P_{-n} f \rightarrow f \quad \text { and } \quad P_{n} f \rightarrow 0 \quad \text { as } n \rightarrow \infty,
$$

with respect to the strong operator topology. Let $K(x, y)$ be as in (4.11), then $P_{n}$ is the integral operator with kernel $2^{-n} K\left(2^{-n} x, 2^{-n} y\right)$. It follows with Lemma 4.1 that $P_{n} f \rightarrow f$ as $n \rightarrow-\infty$. The kernel of $P_{n}$ satisfies

$$
\left|2^{-n} K\left(2^{-n} x, 2^{-n} y\right)\right| \leq \frac{C 2^{-n}}{\left(1+2^{-n}|x-y|\right)^{2}}
$$

and from this inequality we easily derive that $P_{n} f \rightarrow 0$ as $n \rightarrow \infty$. This concludes the proof of Theorem 4.4. 
Example 4.7. The Haar wavelet basis

Let $h_{0}=h_{1}=\frac{1}{2} \sqrt{2}$, then $H(\xi)=\left(1+e^{-i \xi}\right) / 2$, and

$$
H\left(\frac{\xi}{2}\right) H\left(\frac{\xi}{4}\right) \cdots H\left(\frac{\xi}{2^{k}}\right)=\frac{1-e^{-i \xi}}{2^{k}\left(1-e^{-i 2^{-k} \xi}\right)},
$$

and this expression converges towards

$$
\widehat{\phi}(\xi)=\frac{1-e^{-i \xi}}{i \xi}=\int_{0}^{1} e^{-i x \xi} d x .
$$

In other words, $\phi$ is the characteristic function of the interval $[0,1]$. Note that indeed

$$
\phi\left(\frac{x}{2}\right)=\phi(x)+\phi(x-1)
$$

(see also Figure 1). In this case we have $H(\xi)=e^{-\frac{1}{2} i \xi} \cos \frac{1}{2} \xi$ and $H(\xi+\pi)=$ $i e^{-\frac{1}{2} i \xi} \sin \frac{\xi}{2}$, yielding that $|H(\xi)|^{2}+|H(\xi+\pi)|^{2}=1$ (cf. (4.6)).

In the second part of Theorem 4.4 the regularity of $\phi$ has to be assumed explicitly as it did not follow automatically from the assumptions on $h_{k}$. The following result, due to Daubechies [1, p. 949] gives sufficient conditions for the regularity of $\phi$ in terms of the coefficients $h_{k}$.

Proposition 4.8. Let $H(\xi)=\left[\frac{1}{2}\left(1+e^{i \xi}\right)\right]^{N} F(\xi)$ with $F(\xi)=\sum_{k=-\infty}^{\infty} f_{k} e^{-i k \xi}$ such that the following conditions are satisfied:

$$
\begin{gathered}
\sum_{k=-\infty}^{\infty}\left|f_{k}\right| \cdot|k|^{\varepsilon}<\infty \quad \text { for some } \varepsilon>0 \\
|F(\xi)| \leq 2^{K}, \quad \xi \in \mathbb{R},
\end{gathered}
$$

for some $K \in \mathbb{R}$. There is a constant $C>0$ such that

$$
\left|\prod_{k=1}^{\infty} H\left(2^{-k} \xi\right)\right| \leq C(1+|\xi|)^{-N+K} .
$$

\section{$\S 5$ Orthonormal wavelet basis}

Assume that the spaces $V_{n}, n \in \mathbb{Z}$ form a multiresolution analysis of $L^{2}(\mathbb{R})$ with $\phi$ the scaling function. Let $W_{n}$ be the orthogonal complement of $V_{n}$ in $V_{n-1}$, in other words,

$$
V_{n} \oplus W_{n}=V_{n-1}
$$

We prove the following theorem. 
Theorem 5.1. There exists a function $\psi \in W_{0}$, called the mother wavelet, such that $\{\psi(\cdot-k) \mid k \in \mathbb{Z}\}$ forms an orthonormal basis of $W_{0}$. If $\phi$ is regular then $\psi$ is regular as well.

We use the following convention. If $V$ is a subspace of $L^{2}(\mathbb{R})$ then we denote by $\widehat{V}$ the subspace of $L^{2}(\mathbb{R})$ consisting of all Fourier transforms of functions in $V$.

From the fact that $V_{1} \oplus W_{1}=V_{0}$ we conclude that $\widehat{V}_{1} \oplus \widehat{W}_{1}=\widehat{V}_{0}$, that is, $\widehat{W}_{1}$ is the orthogonal complement of $\widehat{V}_{1}$ in $\widehat{V}_{0}$. We have seen that

$$
\begin{aligned}
& \widehat{V}_{0}=\left\{A \widehat{\phi} \mid A \in L_{2 \pi}^{2}\right\} \\
& \widehat{V}_{1}=\left\{A(2 \cdot) \widehat{\phi}(2 \cdot) \mid A \in L_{2 \pi}^{2}\right\} .
\end{aligned}
$$

Using that $\widehat{\phi}(2 \xi)=H(\xi) \widehat{\phi}(\xi)$ (cf. (4.4)) we get that

$$
\widehat{V}_{1}=\left\{A(2 \cdot) H \widehat{\phi} \mid A \in L_{2 \pi}^{2}\right\} .
$$

Let $S: \widehat{V}_{0} \rightarrow L^{2}[0,2 \pi]$ be the unitary operator given by

$$
S(A \widehat{\phi})=A .
$$

Instead of computing $\widehat{W}_{1}$ directly, we first compute $S\left(\widehat{W}_{1}\right)$, which is the orthogonal complement of $S\left(\widehat{V}_{1}\right)$ in $L^{2}[0,2 \pi]$. It is obvious that

$$
S\left(\widehat{V}_{1}\right)=\left\{A(2 \cdot) H \mid A \in L^{2}[0,2 \pi]\right\} .
$$

Let $F \in L^{2}[0,2 \pi]$ be in the orthogonal complement of $S\left(\widehat{V}_{1}\right)$, then we have

$$
\int_{0}^{2 \pi} A(2 \xi) H(\xi) \bar{F}(\xi) d \xi=0,
$$

for every $2 \pi$-periodic function $A$. This means that

$$
\int_{0}^{\pi} A(2 \xi)[H(\xi) \bar{F}(\xi)+H(\xi+\pi) \bar{F}(\xi+\pi)] d \xi=0
$$

for every $2 \pi$-periodic function $A$. But this implies that

$$
H(\xi) \bar{F}(\xi)+H(\xi+\pi) \bar{F}(\xi+\pi)=0 .
$$

So for every $\xi \in \mathbb{R}$, the vector $(F(\xi), F(\xi+\pi))$ is orthogonal with respect to the unit vector $(H(\xi), H(\xi+\pi))$ in the vector space $\mathbb{C}^{2}$. Then there is a function $\alpha$ such that

$$
\begin{aligned}
F(\xi) & =\alpha(\xi) e^{-i(\xi+\pi)} \bar{H}(\xi+\pi) \\
F(\xi+\pi) & =-\alpha(\xi) e^{-i(\xi+\pi)} \bar{H}(\xi) .
\end{aligned}
$$


It follows immediately that $\alpha$ is $\pi$-periodic. Using (4.6) we find that

$$
\alpha(\xi)=e^{i(\xi+\pi)}[F(\xi) H(\xi+\pi)-F(\xi+\pi) H(\xi)] .
$$

From (5.3) we obtain that

$$
\begin{aligned}
\int_{0}^{2 \pi}|F(\xi)|^{2} d \xi & =\int_{0}^{2 \pi}|\alpha(\xi)|^{2}|H(\xi+\pi)|^{2} d \xi \\
& =\int_{0}^{\pi}|\alpha(\xi)|^{2}\left[|H(\xi)|^{2}+|H(\xi+\pi)|^{2}\right] d \xi \\
& =\int_{0}^{\pi}|\alpha(\xi)|^{2} d \xi
\end{aligned}
$$

from which we conclude that the linear operator $F \rightarrow \alpha$ given by (5.5) defines an isometry from $L^{2}[0,2 \pi]$ into $L^{2}[0, \pi]$. This yields that

$$
\pi^{-\frac{1}{2}} e^{-i(\xi+\pi)} \bar{H}(\xi+\pi) e^{2 k i \xi}, \quad k \in \mathbb{Z},
$$

forms an orthonormal basis in $S\left(\widehat{W}_{1}\right)$. Applying $S^{-1}$ we find that

$$
\pi^{-\frac{1}{2}} e^{-i(\xi+\pi)} \bar{H}(\xi+\pi) \widehat{\phi}(\xi) e^{2 k i \xi}, \quad k \in \mathbb{Z},
$$

defines an orthonormal basis of $\widehat{W}_{1}$. Let. $\Psi \in W_{1}$ be the function with Fourier transform

$$
\widehat{\Psi}(\xi)=\sqrt{2} e^{-i(\xi+\pi)} \bar{H}(\xi+\pi) \widehat{\phi}(\xi),
$$

then the family $\{\Psi(\cdot-2 k) \mid k \in \mathbb{Z}\}$ is an orthonormal basis of $W_{\mathbf{1}}$. Let $\psi \in W_{0}$ be given by $\psi=D_{\frac{1}{2}} \Psi$. Then $\widehat{\psi}(2 \xi)=2^{-\frac{1}{2}} \widehat{\Psi}(\xi)$, that is,

$$
\widehat{\psi}(2 \xi)=e^{-i(\xi+\pi)} \bar{H}(\xi+\pi) \widehat{\phi}(\xi),
$$

thus $\{\psi(\cdot-k) \mid k \in \mathbb{Z}\}$ is an orthonormal basis of $W_{0}$. Using that $e^{i \xi} \widehat{\psi}(2 \xi)$ is the Fourier transform of $\frac{1}{2} \psi((x+1) / 2)$ and that

$$
\bar{H}(\xi+\pi) \widehat{\phi}(\xi)=2^{-\frac{1}{2}} \sum_{k=-\infty}^{\infty}(-1)^{k} \bar{h}_{k} e^{i k \xi} \widehat{\phi}(\xi)
$$

we get, by taking the inverse Fourier transform of (5.8), that

$$
\psi\left(\frac{x+1}{2}\right)=\sqrt{2} \sum_{k=-\infty}^{\infty}(-1)^{k+1} \bar{h}_{k} \phi(x+k),
$$


or in other words

$$
\psi(x)=\sqrt{2} \sum_{k=-\infty}^{\infty}(-1)^{k} \bar{h}_{-k+1} \phi(2 x-k) .
$$

We observe that the assertion in Theorem 5.1 about the regularity of $\psi$ follows immediately from the series expansion (5.9).

In the case of the Haar wavelet (see Example 4.7) we have $h_{0}=h_{1}=\frac{1}{2} \sqrt{2}$. This yields that $\psi(x)=\phi(2 x)-\phi(2 x-1)$; (see also Example 1.1).

The system $\left\{\psi_{n, k} \mid k \in \mathbb{Z}\right\}$ with $\psi_{n, k}$ given by

$$
\psi_{n, k}(x)=2^{-\frac{n}{2}} \psi\left(2^{-n} x-k\right),
$$

defines an orthonormal basis of $W_{n}$. Moreover, we can prove the following important theorem.

Theorem 5.2. The system $\left\{\psi_{n, k} \mid k, n \in \mathbb{Z}\right\}$ is an orthonormal basis of $L^{2}(\mathbb{R})$.

This system is called the orthonormal wavelet basis and $\psi$ is called the mother wavelet. To prove this theorem we make the following observations. From the fact that $W_{n} \perp V_{n}$ and $W_{n} \oplus V_{n}=V_{n-1}$ it follows immediately that the $W_{n}$ are mutually orthogonal. Let $Q_{n}$ be the orthogonal projection on $W_{n}$. From

$$
P_{n}=P_{n+1}+Q_{n+1}
$$

we derive that, for $m>n$,

$$
P_{n} f=P_{m} f+\sum_{j=n+1}^{m} Q_{j} f .
$$

Using that $P_{m} f \rightarrow 0$ as $m \rightarrow \infty$ we get that

$$
P_{n} f=\sum_{j=n+1}^{\infty} Q_{j} f
$$

Furthermore, since $P_{n} f \rightarrow f$ as $n \rightarrow-\infty$, we find that

$$
\sum_{n=-\infty}^{\infty} Q_{n} f=f
$$

which proves Theorem 5.2. Instead of (5.12) we can also write

$$
\bigoplus_{n=-\infty}^{\infty} W_{n}=L^{2}(\mathbb{R})
$$


$\S 6$ Wavelet expansion and filtering

We can reformulate (5.6) as

$$
\widehat{\psi}(2 \xi)=G(\xi) \widehat{\phi}(\xi)
$$

where

$$
G(\xi):=e^{-i(\xi+\pi)} \bar{H}(\xi+\pi) .
$$

Let the coefficients $g_{k}$ be defined by

$$
G(\xi)=\frac{1}{\sqrt{2}} \sum_{k=-\infty}^{\infty} g_{k} e^{-i k \xi},
$$

or in other words

$$
g_{k}=(-1)^{k} \bar{h}_{-k+1} .
$$

By taking the inverse Fourier transform of (6.1) we get that

$$
\psi\left(\frac{x}{2}\right)=\sqrt{2} \sum_{k=-\infty}^{\infty} g_{k} \phi(x-k) .
$$

Consider a multiresolution analysis $\left\{V_{n}\right\}$ with scaling function and mother wavelet respectively given by $\phi$ and $\psi$. Let as before

$$
\begin{aligned}
& \phi_{n, k}(x)=2^{-\frac{n}{2}} \phi\left(2^{-n} x-k\right), \\
& \psi_{n, k}(x)=2^{-\frac{n}{2}} \psi\left(2^{-n} x-k\right) .
\end{aligned}
$$

The projections $P_{n}$ on $V_{n}$ and $Q_{n}$ on $W_{n}$ are respectively given by

$$
\begin{aligned}
& P_{n} f=\sum_{k=-\infty}^{\infty} c_{k}^{n}(f) \phi_{n, k}, \\
& Q_{n} f=\sum_{k=-\infty}^{\infty} d_{k}^{n}(f) \psi_{n, k},
\end{aligned}
$$

where

$$
c_{k}^{n}(f)=\left\langle f, \phi_{n, k}\right\rangle \quad \text { and } \quad d_{k}^{n}(f)=\left\langle f, \psi_{n, k}\right\rangle .
$$

Suppose that $P_{n} f$ is known through its coefficients $c_{k}^{n}$. We demonstrate how one can express the coefficients $c_{k}^{n+1}$ of $P_{n+1} f$ and $d_{k}^{n+1}$ of $Q_{n+1} f$ in terms of the $c_{k}^{n}$. From

$$
\phi\left(\frac{x}{2}\right)=\sqrt{2} \sum_{k=-\infty}^{\infty} h_{k} \phi(x-k)
$$


(cf. (4.1)) it follows easily that

$$
\phi_{n, k}=\sum_{l=-\infty}^{\infty} h_{l-2 k} \phi_{n-1, l} .
$$

Let $\mathbf{H}: \ell^{2} \rightarrow \ell^{2}$ (where $\ell^{2}=\ell^{2}(\mathbb{Z})$ ) be the filter given by

$$
(\mathbf{H} a)_{k}=\sum_{l=-\infty}^{\infty} \bar{h}_{l-2 k} a_{l} .
$$

We use the notational convention that

$$
\left(c^{n}\right)_{k}=c_{k}^{n}
$$

Then we obtain from (6.10) and (6.11) that

$$
c^{n}=\mathrm{H} c^{n-1} \text {. }
$$

With (5.7) and (6.4) we derive that

$$
\psi_{n, k}=\sum_{l=-\infty}^{\infty} g_{l-2 k} \phi_{n-1, l} .
$$

Defining the filter $\mathbf{G}$ by

$$
(\mathbf{G} a)_{k}=\sum_{l=-\infty}^{\infty} \bar{g}_{l-2 k} a_{l}
$$

we derive that

$$
d^{n}=\mathrm{G} c^{n-1}
$$

From (6.11) and (6.14) we deduce that for every $n \in \mathbb{Z}$,

$$
\begin{aligned}
\left\langle\phi_{n, l}, \phi_{n-1, k}\right\rangle & =h_{k-2 l}, \\
\left\langle\psi_{n, l}, \phi_{n-1, k}\right\rangle & =g_{k-2 l} .
\end{aligned}
$$

If $\mathbf{F}$ is a filter on $\ell^{2}$, then the adjoint filter is the mapping $\mathrm{F}^{*}: \ell^{2} \rightarrow \ell^{2}$ which satisfies $\langle\mathbf{F} a, b\rangle=\left\langle a, \mathbf{F}^{*} b\right\rangle$. The adjoint filters $\mathbf{H}^{*}, \mathbf{G}^{*}: \ell^{2} \rightarrow \ell^{2}$ are respectively given by

$$
\begin{aligned}
\left(\mathbf{H}^{*} a\right)_{k} & =\sum_{l=-\infty}^{\infty} h_{k-2 l} a_{l}, \\
\left(\mathbf{G}^{*} a\right)_{k} & =\sum_{l=-\infty}^{\infty} g_{k-2 l} a_{l} .
\end{aligned}
$$


Now we can express $c^{n-1}$ in terms of $c^{n}$ and $d^{n}$. We use (6.17)-(6.18) to derive

$$
\begin{aligned}
c_{k}^{n-1} & =\left\langle f, \phi_{n-1, k}\right\rangle \\
& =\left\langle P_{n-1} f, \phi_{n-1, k}\right\rangle \\
& =\left\langle P_{n} f+Q_{n} f, \phi_{n-1, k}\right\rangle \\
& =\left\langle\sum_{l=-\infty}^{\infty} c_{l}^{n} \phi_{n, l}, \phi_{n-1, k}\right\rangle+\left\langle\sum_{l=-\infty}^{\infty} d_{l}^{n} \psi_{n, l}, \phi_{n-1, k}\right\rangle \\
& =\sum_{l=-\infty}^{\infty} h_{k-2 l} c_{l}^{n}+\sum_{l=-\infty}^{\infty} g_{k-2 l} d_{l}^{n} .
\end{aligned}
$$

In other words

$$
c^{n-1}=\mathbf{H}^{*} c^{n}+\mathbf{G}^{*} d^{n}
$$

Remark. 6.1. The transition $c^{n-1} \rightarrow c^{n}, d^{n}$ corresponds to a change of basis in $V_{n-1}$, namely

$$
\left\{\phi_{n-1, k} \mid k \in \mathbb{Z}\right\} \longrightarrow\left\{\phi_{n, k} \mid k \in \mathbb{Z}\right\} \cup\left\{\psi_{n, k} \mid k \in \mathbb{Z}\right\}
$$

The decomposition of the signal $c^{n-1}$ at the level $n-1$ into a lower resolution signal $c^{n}$ and a difference (or detail) signal $d^{n}$ forms the basis for the pyramid algorithm described in [6].

Note that by $(6.2)-(6.3)$,

$$
\frac{1}{\sqrt{2}} \sum_{k=-\infty}^{\infty} g_{k}=G(0)=-\bar{H}(\pi)
$$

From (4.6) and (4.8) we conclude that $|H(\pi)|^{2}=0$, hence

$$
\sum_{k=-\infty}^{\infty} g_{k}=0
$$

Combination of (6.13), (6.16), and (6.21) yields that

$$
\left(\mathbf{H}^{*} \mathbf{H}+\mathbf{G}^{*} \mathbf{G}\right) c^{n-1}=c^{n-1},
$$

from which we conclude that

$$
\mathbf{H}^{*} \mathbf{H}+\mathbf{G}^{*} \mathbf{G}=\mathbf{I d}
$$

In terms of the Fourier transform this relation corresponds to the identity (4.6), i.e.,

$$
|H(\xi)|^{2}+|H(\xi+\pi)|^{2}=1 .
$$


Note that both $\mathbf{H}^{*} \mathbf{H}$ and $\mathbf{G}^{*} \mathbf{G}$ define projection operators. We compute $\mathbf{H H}^{*}$. Let $a \in \ell^{2}$, then

$$
\left(\mathrm{HH}^{*} a\right)_{k}=\sum_{m=-\infty}^{\infty} \sum_{l=-\infty}^{\infty} \bar{h}_{l-2 k} h_{l-2 m} a_{m}=a_{k}
$$

by (4.7). This yields that

$$
\mathbf{H H}^{*}=\mathbf{I d}
$$

Similarly we find that

$$
\mathbf{G G}^{*}=\mathbf{I d},
$$

and

$$
\mathbf{H G}^{*}=\mathbf{G H}^{*}=\mathbf{0} .
$$

$\mathbf{H}$ and $\mathbf{G}$ are called quadrature mirror filters in the literature.

An alternative proof of the identities (6.24)-(6.26) can be given by exploiting the decomposition of $V_{0}$ into the orthogonal subspaces $V_{1}$ and $W_{1}$. Let $c, d \in \ell^{2}$ be arbitrary and define $f \in V_{0}$ by

$$
f=\sum_{k=-\infty}^{\infty} c_{k} \phi_{1, k}+\sum_{k=-\infty}^{\infty} d_{k} \psi_{1, k}
$$

The first term at the right hand-side lies in $V_{1}$ whereas the second term lies in $W_{1}$. Alternatively, we can write

$$
f=\sum_{k=-\infty}^{\infty} \tilde{c}_{k} \phi_{0, k}
$$

where $\tilde{c}=\mathbf{H}^{*} c+\mathbf{G}^{*} d$. Now

$$
c=\mathbf{H} \tilde{c}=\mathbf{H H}^{*} c+\mathbf{H G}^{*} d \quad \text { and } \quad d=\mathbf{G} \tilde{c}=\mathbf{G H}^{*} c+\mathbf{G G}^{*} d .
$$

Since these identities hold for arbitrary $c, d \in \ell^{2}$, relations (6.24)- (6.26) follow.

\section{$\S 7$ The sinc-wavelet and the Meyer wavelet}

In the present section we discuss two examples of a wavelet basis for which the Fourier transform of both the scaling function $\phi$ and the wavelet function $\psi$ has a compact support.

The sinc-wavelet

The first example is the so-called sinc-wavelet. Recall that sinc is a well-known function in signal analysis given by $\operatorname{sinc}(x)=\sin x / x$; (see Figure 3 ). It is easy to 

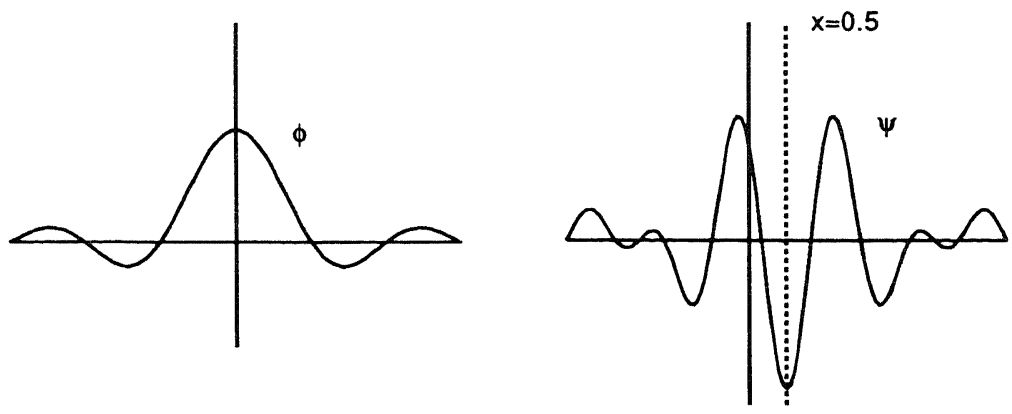

Figure 3. The scaling function $\phi(x)=\operatorname{sinc}(\pi x)$ (left) and the corresponding wavelet function $\psi$ (right).

check that the Fourier transform of $\operatorname{sinc}(\pi x)$ is the characteristic function of the interval $[-\pi, \pi]$.

Let $V_{0}$ be the subspace of $L^{2}(\mathbb{R})$ given by

$$
V_{0}=\left\{f \in L^{2}(\mathbb{R}) \mid \operatorname{supp}(\widehat{f}) \subseteq[-\pi, \pi]\right\}
$$

where $\operatorname{supp}(f)$ is the support of the function $f$. To see that (M4) is satisfied, take $f \in V_{0}$. Since the Fourier transform of $f(\cdot-k)$ is given by $e^{-i k \cdot} \hat{f}$ it is clear that $f(\cdot-k) \in V_{0}$. Let $V_{n}$ be the space deriving from $V_{0}$ by dilation. Then

$$
V_{n}=\left\{f \in L^{2}(\mathbb{R}) \mid \operatorname{supp}(\widehat{f}) \subseteq\left[-2^{-n} \pi, 2^{-n} \pi\right]\right\} .
$$

Therefore (M1) is trivially satisfied. The projection $P_{n}$ is given by

$$
P_{n} f(x)=\frac{1}{2 \pi} \int_{-2^{-n} \pi}^{2^{-n} \pi} \widehat{f}(\xi) e^{i x \xi} d \xi
$$

and from this expression one easily derives that $P_{n} f \rightarrow f$ as $n \rightarrow-\infty$. Therefore (M2) holds as well.

We show that $\phi(x)=\operatorname{sinc}(\pi x)$ is a scaling function, that means, the collection $\phi(\cdot-k), k \in \mathbb{Z}$ constitutes an orthonormal basis of $V_{0}$. To prove this observe that the Fourier transform of $x \rightarrow \operatorname{sinc}(\pi x)$ is the characteristic function of the interval $[-\pi, \pi]$. This yields that

$$
\int_{\mathbb{R}} \operatorname{sinc}(\pi x) \operatorname{sinc}(\pi(x-k)) d x=\frac{1}{2 \pi} \int_{-\pi}^{\pi} e^{i k \xi} d \xi=\delta_{k 0},
$$

from which the assertion follows. Considering $x$ as a complex variable $V_{0}$ becomes the Paley-Wiener space consisting of entire functions of exponential type at most 
$\pi[10$, p. $105 \mathrm{ff}]$. For $f \in V_{0}$ we have

$$
\|f\|^{2}=\sum_{k=-\infty}^{\infty}|f(k)|^{2}
$$

and

$$
f(x)=\sum_{k=-\infty}^{\infty} f(k) \operatorname{sinc}(\pi(x-k)) .
$$

The latter series is called the cardinal series and plays an important role in signal analysis where it is known as the sampling theorem.

The coefficients $h_{k}$ are determined by the relation

$$
\phi\left(\frac{x}{2}\right)=\sqrt{2} \sum_{k=-\infty}^{\infty} h_{k} \phi(x-k),
$$

and we find that

$$
h_{0}=\frac{1}{\sqrt{2}}, \quad h_{2 k}=0 \text { if } k \neq 0, \quad h_{2 k+1}=\sqrt{2} \frac{(-1)^{k}}{\pi(2 k+1)} .
$$

The $2 \pi$-periodic function $H(\xi)=2^{-\frac{1}{2}} \sum_{k=-\infty}^{\infty} h_{k} e^{-i k \xi}$ can easily be found from the relation

$$
\widehat{\phi}(2 \xi)=H(\xi) \widehat{\phi}(\xi),
$$

and the fact that $\hat{\phi}$ is the characteristic function of the interval $[-\pi, \pi]$.

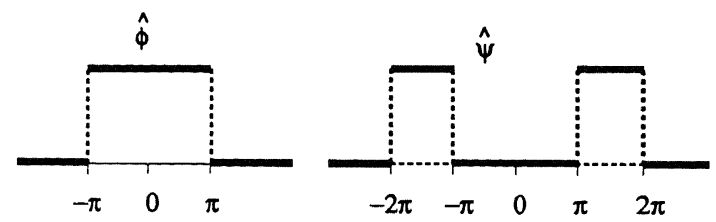

Figure 4. The functions $\widehat{\phi}$ and $\widehat{\psi}$.

The Fourier transform $\widehat{\psi}$ of the corresponding wavelet function $\psi$ is given by

$$
\widehat{\psi}(\xi)=G\left(\frac{\xi}{2}\right) \widehat{\phi}\left(\frac{\xi}{2}\right)
$$

where $G$ is related to $H$ through (6.2). Thus we find that $\widehat{\psi}(\xi)=e^{-i(\xi / 2+\pi)}$ if $\pi \leq|\xi| \leq 2 \pi$ and 0 elsewhere; (see Figure 4). Since $H=0$ in a neighbourhood of 
$\pi$ we find that $G^{(r)}(0)=0$ for every integer $r \geq 0$. This yields that $\widehat{\psi}^{(r)}(0)=0$ for $r \geq 0$, and therefore

$$
\int_{\mathbf{R}} x^{r} \psi(x) d x=0 \quad \text { for every } r \geq 0
$$

In this example $\psi$ can be computed explicitly as the inverse Fourier transform of $\widehat{\psi}$. A straightforward computation shows that

$$
\psi(x)=\frac{\cos \pi x-\sin 2 \pi x}{\pi\left(\frac{1}{2}-x\right)} .
$$

Note that $\psi$ is symmetric around $x=\frac{1}{2}$, that is $\psi(x)=\psi(1-x)$; (see Figure 3 ).

The Meyer wavelet

The second example which we discuss in this section is closely related to the previous one. This example was first reported by Yves Meyer and for that reason it is often called the Meyer wavelet; however, Meyer called it the Littlewood-Paley wavelet. In fact, the main difference between this example and the sinc-wavelet discussed above is that the Fourier transform of the Meyer wavelet has arbitrary high regularity.

We denote by $\mathcal{D}(\mathbb{R})$ the space of all functions which have compact support and are infinitely many times differentiable. By $\mathcal{S}(\mathbb{R})$ we denote the class of functions $f$ which are infinitely many times differentiable and satisfy the estimates

$$
\left|f^{(p)}(x)\right|=\mathcal{O}\left(|x|^{-n}\right), \quad|x| \rightarrow \infty,
$$

for all nonnegative integers $p, n$. It is obvious that $\mathcal{D}(\mathbb{R}) \subseteq \mathcal{S}(\mathbb{R})$. Furthermore it can be shown that

$$
f \in \mathcal{S}(\mathbb{R}) \Longleftrightarrow \widehat{f} \in \mathcal{S}(\mathbb{R}) .
$$

The wavelet $\psi$ which we construct below will have the following properties:

- $\widehat{\psi}$ has finite support

- $\psi, \widehat{\psi} \in \mathcal{S}(\mathbb{R})$

- $\psi(x)=\psi(1-x)$, for $x \in \mathbb{R}$

- $\int_{\mathbf{R}} x^{p} \psi(x) d x=0$ for every integer $p \geq 0$.

A comprehensive discussion on the Meyer wavelet can also be found in [7] and [3]. We start by choosing a function $P$ with the following properties:

(i) $P \in \mathcal{D}(\mathbb{R})$

(ii) $P$ is even, i.e., $P(\xi)=P(-\xi)$

(iii) $0 \leq P \leq 1$

(iv) $P$ has finite support, to be precise $\operatorname{supp}(P)=(-4 \pi / 3,4 \pi / 3)$

(v) $P(\xi)=1$ for $\xi \in[-2 \pi / 3,2 \pi / 3]$

(vi) $P(\xi)^{2}+P(2 \pi-\xi)^{2}=1$ for $0 \leq \xi \leq 2 \pi$. 


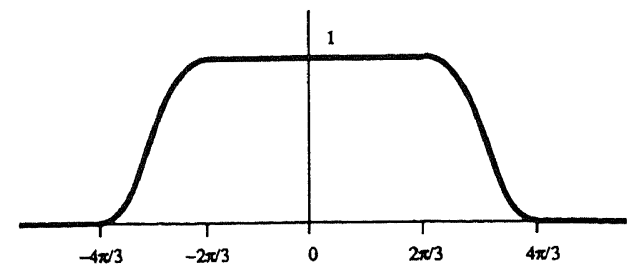

Figure 5. The function $\widehat{\phi}=P$ used in the construction of the Meyer wavelet.

Thus $P$ is a function with a shape as depicted in Figure 5. At the end of this section we shall make a short remark concerning the construction of such a function $P$.

It is easy to verify that for every $k \in \mathbb{Z}$,

$$
\int_{\mathbf{R}} e^{-i k \xi} P(\xi)^{2}=2 \pi \cdot \delta_{k 0} .
$$

There exists a function $\phi \in \mathcal{S}(\mathbb{R})$ with Fourier transform

$$
\widehat{\phi}=P \text {. }
$$

Then

$$
\begin{aligned}
\int_{\mathbf{R}} \phi(x) \bar{\phi}(x-k) d x & =\frac{1}{2 \pi} \int_{\mathbf{R}} e^{i k \xi}|\widehat{\phi}(\xi)|^{2} d \xi \\
& =\frac{1}{2 \pi} \int_{\mathbf{R}} e^{i k \xi}|P(\xi)|^{2} d \xi=\delta_{k 0} .
\end{aligned}
$$

Thus $\{\phi(\cdot-k) \mid k \in \mathbb{Z}\}$ is an orthonormal system. Let $V_{0}$ be the space spanned by this system and let $V_{n}$ be the spaces obtained by dilation. Then $\widehat{V}_{0}=\{A \widehat{\phi} \mid A \in$ $\left.L_{2 \pi}^{2}\right\}$ and $\widehat{V}_{-1}=\left\{A \widehat{\phi}\left(\frac{1}{2} \cdot\right) \mid A \in L_{4 \pi}^{2}\right\}$. We show that $V_{0} \subset V_{-1}$, that is, $\widehat{V}_{0} \subset \widehat{V}_{-1}$. Let $H$ be the $2 \pi$-periodic function (see Figure 6).

$$
H(\xi)=P(2 \xi), \quad \xi \in[-\pi, \pi] .
$$

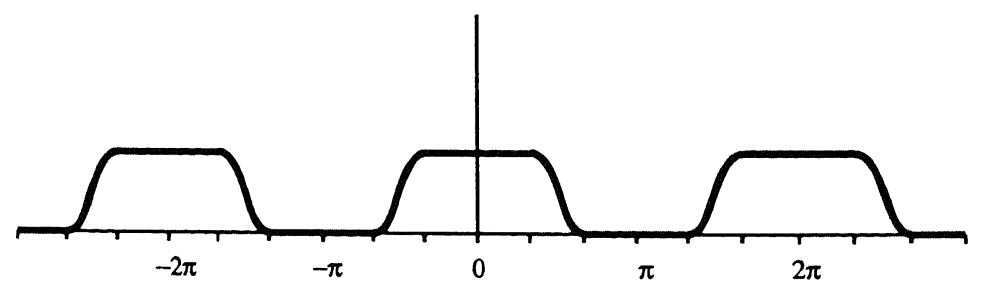

Figure 6. The periodic function $H$. 
Then

$$
\widehat{\phi}(2 \xi)=H(\xi) \widehat{\phi}(\xi) .
$$

To understand this one should observe that $\widehat{\phi}(\xi)=1$ for every $\xi$ in the support of $\widehat{\phi}(2 \cdot)$. With (7.3) it follows easily that $\widehat{V}_{0} \subset \widehat{V}_{-1}$. Using property (v) of $P$ it is easy to prove that

$$
|H(\xi)|^{2}+|H(\xi+\pi)|^{2}=1 .
$$

We have thus shown that the conditions of Theorem 4.4 are fulfilled. Note that (4.14) follows from the fact that $H$ is infinitely often differentiable. Let $G(\xi)=$ $e^{-i(\xi+\pi)} \bar{H}(\xi+\pi)$, then

$$
\widehat{\psi}(\xi)=G\left(\frac{\xi}{2}\right) \widehat{\phi}\left(\frac{\xi}{2}\right)=e^{-i\left(\frac{\xi}{2}+\pi\right)} H\left(\frac{\xi}{2}+\pi\right) \widehat{\phi}\left(\frac{\xi}{2}\right) .
$$

A straightforward computation shows that

$$
\operatorname{supp}(\widehat{\psi})=(-8 \pi / 3,-2 \pi / 3) \cup(2 \pi / 3,8 \pi / 3) ;
$$

(see Figure 7).

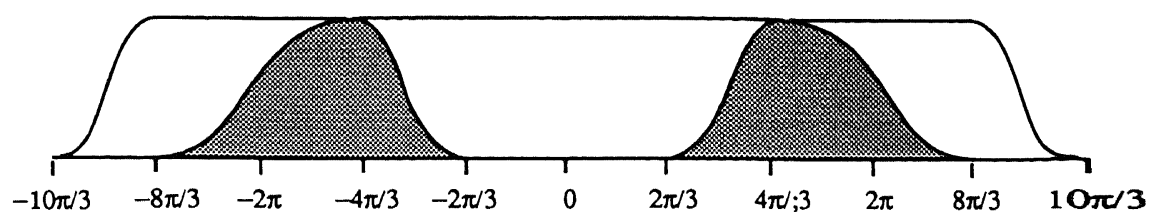

Figure 7. The Fourier transform $\widehat{\psi}$.

Therefore $\widehat{\psi} \in \mathcal{S}(\mathbb{R})$ and thus $\psi \in \mathcal{S}(\mathbb{R})$. We call $\psi$ the Meyer wavelet. From (7.3), (7.4) and the fact that $H(\xi) \geq 0$ everywhere one easily derives that

$$
\widehat{\psi}(\xi)=e^{-i\left(\frac{\xi}{2}+\pi\right)}\left[\widehat{\phi}\left(\frac{\xi}{2}\right)^{2}-\widehat{\phi}(\xi)^{2}\right]^{\frac{1}{2}} .
$$

Note that, just as in the example of the sinc-wavelet, the wavelet transform has the effect of a band-pass filter. It is clear that $e^{i \xi / 2} \widehat{\psi}(\xi)$ is even, and with this observation it is not difficult to show that

$$
\psi(x)=\psi(1-x) .
$$

Finally, since $G^{(p)}(0)=0$ for every integer $p \geq 0$, we get that $\widehat{\psi}^{(p)}(0)=0$ for every $p \geq 0$. Therefore we have

$$
\int_{\mathbf{R}} x^{p} \psi(x) d x=0, \quad p \geq 0
$$


We conclude this example with a remark about the construction of a function $P$ satisfying the properties mentioned at the beginning of this section, in particular the property that $P(\xi)^{2}+P(2 \pi-\xi)^{2}=1$ for every $\xi$. Choose a $C^{\infty}$-function $\alpha$ on $[0,2 \pi]$ with $\alpha=0$ on $[0,2 \pi / 3]$, increasing on $(2 \pi / 3,4 \pi / 3)$ and $\alpha=\pi / 2$ on $[4 \pi / 3,2 \pi]$. Furthermore let $\alpha$ be symmetric with respect to the point $\xi=\pi$, that is

$$
\alpha(2 \pi-\xi)=\frac{\pi}{2}-\alpha(\xi)
$$

(see Figure 8).

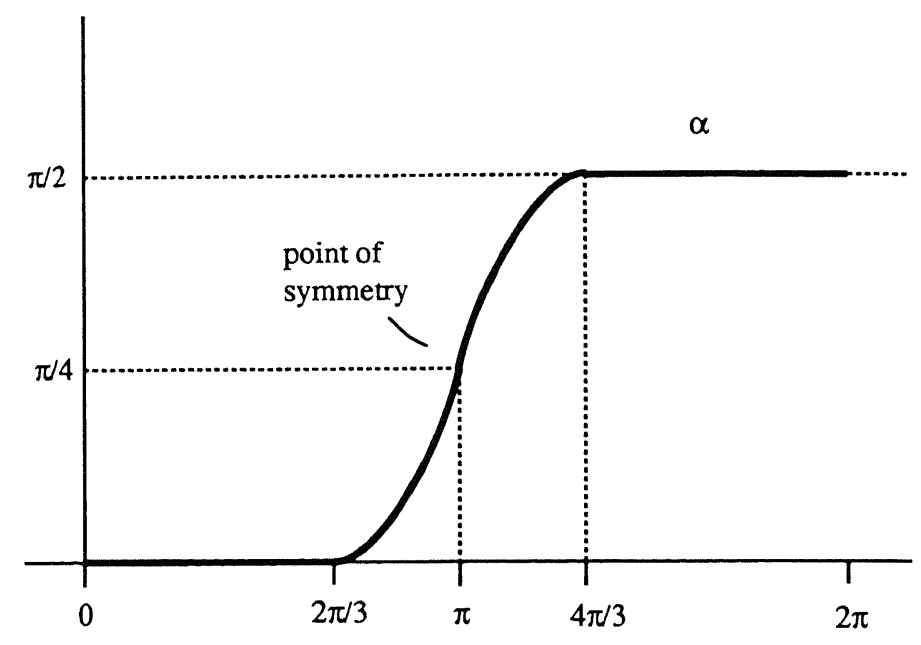

Figure 8. The $C^{\infty}$-function $\alpha$.

Define $P(\xi)=\cos \alpha(\xi)$ for $\xi \in[0,2 \pi]$. Then $P(2 \pi-\xi)=\cos \alpha(2 \pi-\xi)=$ $\cos (\pi / 2-\alpha(\xi))=\sin \alpha(\xi)$, from which it follows immediately that $P(\xi)^{2}+P(2 \pi-$ $\xi)^{2}=1$.

\section{References}

1. Daubechies, I., Orthonormal bases of compactly supported wavelets, Comm. Pure and Appl. Math. 41 (1988), 909-996.

2. David, G. Wavelets and Singular Integrals on Curves and Surfaces, SpringerVerlag, Berlin, 1991.

3. Frazier, M., B. Jawerth, and G. Weiss, Littlewood-Paley Theory and the Study of Function Spaces, CBMS, Regional Conference Series in Mathematics 79, AMS, Providence, 1991. 
4. Heil, C.E. and D.F. Walnut, Continuous and discrete wavelet transforms, SIAM Review 31 (1989), 628-666.

5. Mallat, S.G., Multiresolution approximations and wavelet orthonormal bases of $L^{2}(\mathbb{R})$, Trans. Amer. Math. Soc. 315 (1989), 69-87.

6. Mallat, S.G., A theory for multiresolution signal decomposition: the wavelet representation, IEEE Trans. Pattern Anal. Mach. Intell. 11 (1989), 674-693.

7. Meyer, Y., Ondelettes et Operateurs I, Hermann, Paris, 1990.

8. Rudin, W., Real and Complex Analysis, McGraw-Hill, New York, 1974.

9. Strang, G., Wavelets and dilation equations: a brief introduction, SIAM Review 31 (1989), 614-627.

10. Young, R.M., An Introduction to Nonharmonic Fourier Series, Academic Press, New York, 1980.

Henk J. A. M. Heijmans

CWI

P.O. Box 4079

1009 AB Amsterdam

The Netherlands

henkh@cwi.nl 